\title{
ESTUDIO DEL PERFIL DE HUMEDAD EN MADERA DE Eucalyptus nitens CON EL AUXILIO DEL MICROTOMO ROTATORIO DE DESPLAZAMIENTO HORIZONTAL
}

\author{
Rozas, Carlos ${ }^{4}$; Cofre, Jordana y Muñoz, Freddy
}

\section{RESUMEN}

El presente estudio tiene como objetivo determinar los perfiles de humedad en madera de Eucalyptus nitens con el auxilio del micrótomo manual rotatorio de desplazamiento horizontal.

Se utilizó madera de corte tangencial de $36 \mathrm{~mm}$ de espesor, $100 \mathrm{~mm}$ de ancho y $60 \mathrm{~cm}$ de largo, las cuales fueron sometidas a diferentes temperaturas de secado $\left(40^{\circ} \mathrm{C}, 60^{\circ} \mathrm{C}, 80^{\circ} \mathrm{C}, 100^{\circ} \mathrm{C}\right.$ y $\left.120^{\circ} \mathrm{C}\right)$, en una estufa de laboratorio.

El perfil de humedad fue obtenido al inicio y en el momento que aparecieron las grietas superficiales. Además, se determinó el contenido de humedad, la densidad básica y la densidad anhidra de cada una de las muestras utilizadas en este estudio.

Los resultados obtenidos para la densidad básica, densidad anhidra y contenido de humedad inicial promedio, fue de $0,51 \mathrm{~g} / \mathrm{cm}^{3}, 0,65 \mathrm{~g} / \mathrm{cm}^{3}$ y $117,7 \%$, respectivamente.

De los perfiles de humedad obtenidos, se pudo comprobar que esta madera presenta una rápida pérdida de humedad superficial en cuanto el centro esta cercano al contenido de humedad inicial, concluyéndose que esta madera presenta una baja permeabilidad y que el movimiento de agua que la caracteriza es por difusión.

Se observó, también que existe una estrecha relación entre el contenido de humedad inicial y el contenido de humedad en el cual ocurren las grietas siendo que, cuanto mayor el contenido de humedad inicial más alto es el contenido de humedad en el cual ocurren las grietas superficiales en la madera.

Palabras clave: Secado de madera, Eucalyptus nitens.

${ }^{4}$ Universidad del Bio Bio. Chile erozas@ubiobio.cl 


\section{ABSTRACT}

The present study aims to determine the moisture profiles of Eucalyptus nitens wood with the aid of the manual of horizontal scrolling rotary microtome.

Wood samples were sawed in tangential cut at $36 \mathrm{~mm}$ thick, $100 \mathrm{~mm}$ wide and $60 \mathrm{~cm}$ long, and were dried under different temperatures $\left(40^{\circ} \mathrm{C}, 60^{\circ} \mathrm{C}, 80^{\circ} \mathrm{C}, 100^{\circ} \mathrm{C}\right.$ and $\left.120^{\circ} \mathrm{C}\right)$ in a laboratory oven.

The moisture profile was obtained at the beginning and at the time that appeared surface cracks. In addition, it was determined the moisture content, the basic density and the anhydrous density of each of the samples used in this study.

The results obtained for the basic density, the anhydrous and the initial moisture content average was $0.51 \mathrm{~g} / \mathrm{cm}^{3}, 0.65 \mathrm{~g} / \mathrm{cm}^{3}$ and $117.7 \%$, respectively.

From the moisture profiles obtained, it was possible to verify that this wood presents a surface moisture rapid loss as soon as the center is close to the initial moisture content, and the conclusion is that this wood has a low permeability and that the water movement water is characterized by diffusion.

It was also noted that there is a close relationship between the initial moisture content and the moisture content at which occurs the cracks and the greater the initial moisture content the higher the moisture content in which occur surface cracks in the wood.

Key words: Wood drying, Eucalyptus nitens. 


\section{INTRODUCCION}

La madera de Eucalyptus nitens es una excelente materia prima para la industria de la celulosa y papel, sin embargo para las industrias de transformación mecánica no ha sido fácil la elaboración de productos de mayor valor agregado, debido a limitaciones y características desfavorables, tales como la presencia de las tensiones de crecimiento, contracción relativamente alta, susceptibilidad al colapso durante el secado y principalmente a la presencia de las grietas superficiales e internas. Estas graves distorsiones y degradación en la calidad de la madera aserrada, la convierten en una especie cuya utilización como madera sólida presenta un gran desafío tecnológico.

Según estudios realizados en la Universidad del Bío-Bío la madera de Eucalyptus nitens presenta una fuerte evaporación superficial, generando altos gradientes de humedad, contracciones prematuras a nivel de superficie y tensiones de secado, originando grietas superficiales e internas. Cuando el colapso es severo, debido a la baja resistencia mecánica de la pared celular de la madera, se generan grietas superficiales e internas en la madera (Rozas et al., 2005).

La madera se encuentra con un contenido de humedad uniforme dentro de la pieza solo cuando es recién aserrada, luego que se inicia el proceso de secado esta humedad comienza a modificarse generando un movimiento desde el centro hacia la superficie, desde la zonas de alta humedad hacia las zonas de baja humedad, produciendo así una diferencia de humedad entre el centro y la superficie de la pieza. Esto significa que la parte externa debe estar más seca que el interior de la madera para que ocurra el secado. La diferencia que se define entre el contenido de humedad de la pieza en el centro y en la superficie es lo que se conoce como gradiente de humedad.

La madera verde al momento de ser sometida a un proceso de secado contiene un flujo de agua libre sobre el punto de saturación de las fibras (PSF) y de agua higroscópica y vapor de agua bajo el PSF. El agua libre contenida en los lúmenes de las células se vincula directamente con la propiedad de permeabilidad que posee la madera, mientras que la difusión de agua higroscópica bajo el PSF tiene relación con la densidad de las paredes celulares. Los perfiles de humedad entonces dependen en gran parte de las propiedades físicas de la madera y el control de los factores que afectan el proceso de secado.

Sobre el punto de saturación de las fibras los gradientes de humedad son distintos entre maderas permeables y poco permeables, en el caso de las maderas permeables éstas tienen un mayor flujo de pérdida de agua libre, lo que conlleva a gradientes de humedad menores. En el caso que la humedad de la madera se encuentre bajo el punto saturación de las fibras para maderas permeables y poco permeables los gradientes de humedad son esencialmente los mismos (Hart and Darwin, 1971).

Durante el proceso de secado la humedad de la madera se mueve principalmente sobre el punto de saturación de las fibras, siendo el objetivo fundamental en esta etapa del proceso eliminar el contenido de humedad lo más pronto posible evitando provocar defectos. Cuando se le aplica a la madera un proceso de secado demasiado brusco los gradientes de humedad obtenidos son excesivos y la contracción desigual resultante puede ocasionar defectos en la madera. Al tener la capacidad de predecir los perfiles de humedad se pueden controlar los programas y las condiciones de secado previniendo así los defectos que se producen en este proceso (Resch et al., 1989).

Hawley (1931), Simpson (1976) y Rice and Young (1991) discutieron los mecanismos de secado que existen en la madera. Ellos compararon dos tipos de secado extremo; uno donde no hay movimiento de agua libre (Figura $\mathrm{N}^{\circ} 1 \mathrm{a}$ ) y un segundo mecanismo donde existe movimiento de agua libre hacia la superficie (Figura $\mathrm{N}^{\circ} 1 \mathrm{~b}$ ). 
La Figura $\mathrm{N}^{\circ}$ 1a muestra una madera poco permeable con su respectivo gradiente de humedad y con una pérdida de humedad en sus dos caras en forma simétrica. El movimiento de humedad en la superficie de la pieza ocurre por difusión a causa de un gradiente higroscópico entra la superficie y el centro, y en este caso el contenido de humedad aumenta en una línea vertical. De este modo el agua libre se mueve solo en esta línea húmeda y el contenido de humedad que existe en el centro se mantiene en el nivel de contenido de humedad inicial hasta que se contrae la línea húmeda. Consecuentemente el perfil de humedad resulta ser discontinuo en el PSF.

En la Figura $\mathrm{N}^{\circ} 1 \mathrm{~b}$ se puede observar un mecanismo que combina el movimiento de difusión y agua líquida, a través del gradiente de humedad. El movimiento de agua en este caso sucede tanto en el interior como en la superficie. El perfil de humedad resulta ser continuo y en forma parabólica. No existe línea húmeda y el agua libre puede moverse del centro antes que este alcance el punto de saturación de las fibras. Este tipo de gradiente prevalece en la mitad y en las últimas etapas del proceso de secado.

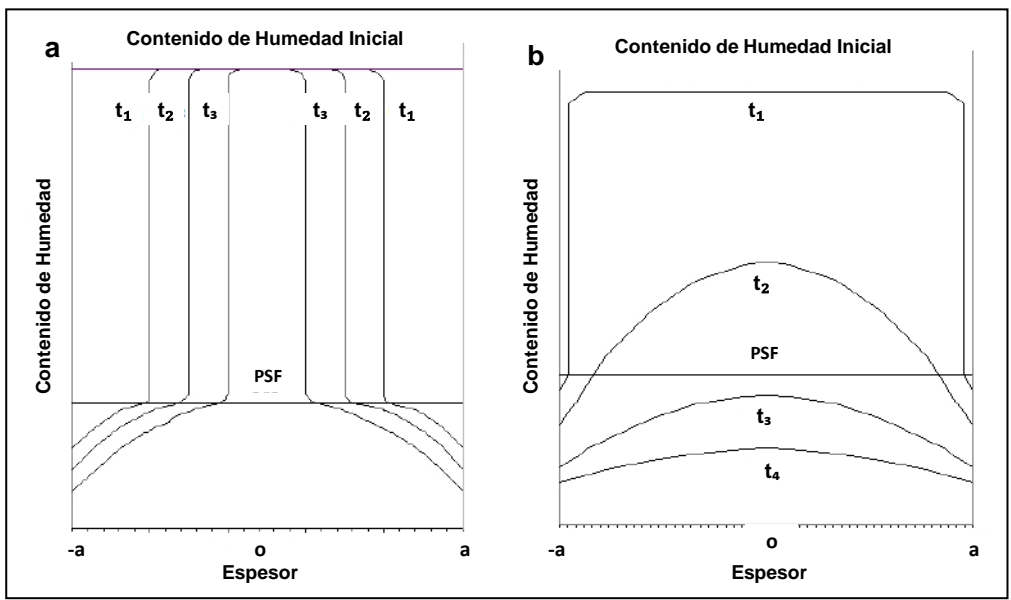

(Fuente: Perfil de humedad según Hawley, 1931. Adaptado de Rice and Young, 1991)

a) madera poco permeable b) madera permeable

$t_{1}, t_{2}, t_{3}, t_{4}:$ tiempo de secado

Figura $\mathrm{N}^{\circ} 1$

EJEMPLOS DE PERFIL DE HUMEDAD

Las grietas superficiales son aberturas en la superficie de la pieza que se deben a los esfuerzos de tracción en la superficie de madera. La aparición de las grietas superficiales acontece cuando el esfuerzo en tracción perpendicular a las fibras excede la resistencia mecánica de la madera (tejidos leñosos), provocando una ruptura en esta. Este tipo de grietas suelen aparecer cuando las condiciones ambientales son muy severas, por ejemplo bajas humedades relativas, producen un secado muy rápido de las capas superficiales, alcanzando valores bajo el PSF, mientras que las capas que se encuentran al interior de la pieza todavía se encuentran con contenido de humedad sobre el $30 \%$.

Normalmente las fisuras aparecen en los tejidos que componen los radios, los cuales son constituidos por las células parenquimáticas (Galvao and Jankowsky, 1985. Citado por De Andrade, 2000). Por lo general, se producen en la etapa inicial del proceso de secado y si se consigue detectarlas a tiempo, se pueden reducir manejando las condiciones ambientales que 
afectan el proceso, tales como la humedad relativa y la temperatura (Rozas, 2007). Según STCP (1990) citado por De Andrade (2000), existen mayores posibilidades que aparezcan grietas en maderas con una alta densidad, las cuales son maderas poco permeables y en maderas gruesas (espesores mayores). El objetivo de este estudio es evaluar el perfil de humedad en madera de Eucalyptus nitens con el auxilio del del micrótomo rotatorio de desplazamiento horizontal.

\section{MATERIAL Y METODO}

En este estudio se utilizó madera aserrada de Eucalyptus nitens en corte tangencial, las dimensiones de las tablas fueron $35 \mathrm{~mm}$ de espesor, $100 \mathrm{~mm}$ de ancho y un metro de largo, que fueron obtenidas de la periferia de trozas con un diámetro de $30 \mathrm{~cm}$ y un metro de largo. De cada tabla fue retirada una muestra de $60 \mathrm{~cm}$ de largo para el estudio de medición del perfil de humedad, tanto al inicio del secado como cuando ocurren las grietas superficiales. Dos muestras de $20 \mathrm{~mm}$ de cada lado para la determinación de la densidad básica y contenido de humedad inicial de la madera de acuerdo a lo establecido en las Normas Chilenas NCh 176/1. Of. 84 y NCh 176/2. Of. 84 (INN, 1986a; 1986b). Para disminuir la pérdida de humedad en el sentido longitudinal, las muestras fueron selladas con pasta selladora y papel aluminio en sus extremos.

Las muestras fueron sometidas a secado en estufa de laboratorio a temperaturas constantes de $40^{\circ} \mathrm{C}, 60^{\circ} \mathrm{C}, 80^{\circ} \mathrm{C}, 100^{\circ} \mathrm{C}$ y $120^{\circ} \mathrm{C}$. La estufa fue precalentada a la temperatura correspondiente antes de comenzar el ensayo de secado. La temperatura de la estufa fue controlada por el propio termómetro de la estufa y por un termómetro adicional colocado dentro de esta. El ensayo finalizaba una vez que las muestras presentaban grietas superficiales, obteniéndose de cada probeta una muestra cercana a la superficie agrietada, en forma de cubo de dimensiones $20 \times 20 \times 36 \mathrm{~mm}$ de la cual fueron obtenidas 35 láminas de $0,7 \mathrm{~mm}$ de espesor a través del micrótomo manual de desplazamiento horizontal, las cuales fueron utilizadas para evaluar el perfil de humedad (Figura $\mathrm{N}^{\circ} 2$ ).

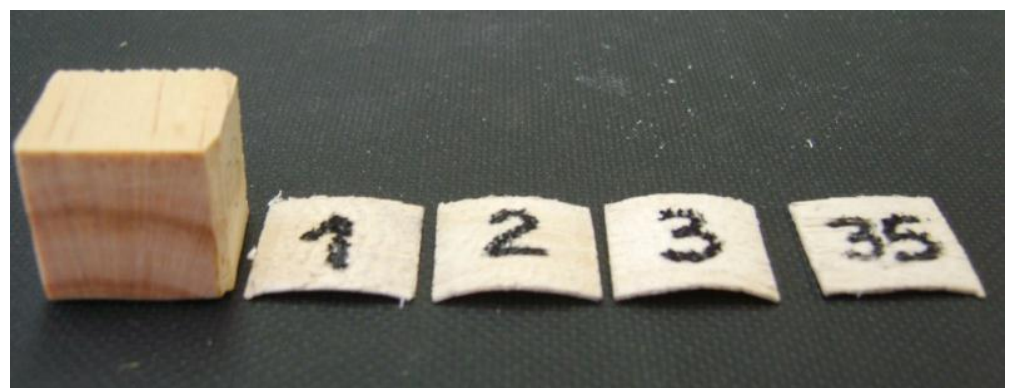

Figura № 2

PROBETAS PARA GRADIENTE DE HUMEDAD

\section{RESULTADO Y DISCUSION}

En el Cuadro № 1 se muestran los valores promedios de la densidad básica y anhidra y el contenido de humedad inicial de todas las probetas utilizadas, encontrándose que la densidad básica de la madera fue de $0,51 \mathrm{~g} / \mathrm{cm}^{3}$, la densidad anhidra de $0,65 \mathrm{~g} / \mathrm{cm}^{3}$ y un contenido de humedad inicial de $117,7 \%$. Estos valores son comparables con los obtenidos por Rozas y Gallardo (2003); densidad básica $0,54 \mathrm{~g} / \mathrm{cm}^{3}$ y contenido de humedad $107 \%$ para la madera de Eucalyptus nitens. 
Cuadro $\mathrm{N}^{\circ} 1$

VALORES PROMEDIO DE DENSIDAD BÁSICA, ANHIDRA

Y CONTENIDO DE HUMEDAD INICIAL DE LA MADERA

\begin{tabular}{|l|c|c|c|}
\hline Parámetros & $\begin{array}{c}\rho_{b} \\
\left(\mathbf{g} / \mathrm{cm}^{3}\right)\end{array}$ & $\begin{array}{c}\rho^{\mathbf{o}} \\
\left(\mathbf{g} / \mathrm{cm}^{3}\right)\end{array}$ & $\begin{array}{c}\text { Chi } \\
(\mathbf{\%})\end{array}$ \\
\hline Probetas $\left(\mathrm{N}^{\circ}\right)$ & 60 & 60 & 60 \\
\hline Promedio & 0,51 & 0,65 & 117,7 \\
\hline Desviación Estándar & 0,06 & 0,0016 & 29,4 \\
\hline Coeficiente Variación & 0,12 & 0,0025 & 0,25 \\
\hline
\end{tabular}

En la Figura № 3 se aprecia la relación que existe entre el contenido de humedad inicial que posee la madera y el contenido de humedad en el momento que se presentan las grietas superficiales. Se observa claramente la tendencia que cuanto mayor el contenido de humedad inicial de la madera el contenido de humedad al cual aparecen las grietas también aumenta. Por ejemplo, para un contenido de humedad inicial entre $80,2 \%$ y $110,1 \%$ el contenido de humedad en que aparecen las grietas fue entre $54,1 \%$ y $91 \%$, y para un con tenido de humedad entre $122,8 \%$ y $158,8 \%$ las grietas superficiales ocurrieron entre $91,8 \%$ y $145,0 \%$.

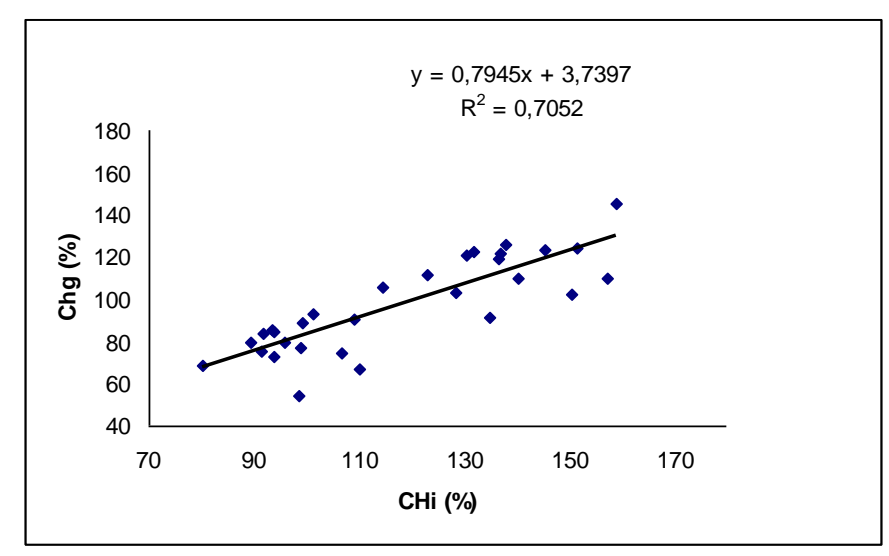

Figura № 3

RELACIÓN ENTRE EL CONTENIDO DE HUMEDAD INICIAL Y EL CONTENIDO DE HUMEDAD CUANDO APARECEN LAS GRIETAS SUPERFICIALES

En las Figuras № $4, N^{\circ} 5, N^{\circ} 6, N^{\circ} 7$ y $N^{\circ} 8$ se pueden observar los perfiles de humedad para cada una de las temepraturas de secado. Cada punto representa el contenido de humedad de cada lámina de $0,7 \mathrm{~mm}$ de espesor. Se puede apreciar, que los valores de contenido de humedad en la superficie cuando aparecen las grietas superficiales, se encuentran cercanos o bajo el PSF, hallándose entre $11,8 \%$ y $31,5 \%$, mientras que en el centro de la pieza el contenido de humedad aún se encuentra sobre el punto de saturación de las fibras, entre 45,2\% y 126,4\%. Las flechas observadas en los gráficos indican la pendiente que representan las capas mas cercanas a la superficie y se puede estimar que mientras mas inclinada se encuentre esta pendiente, acercándose al eje horizontal, el gradiente será menor. 


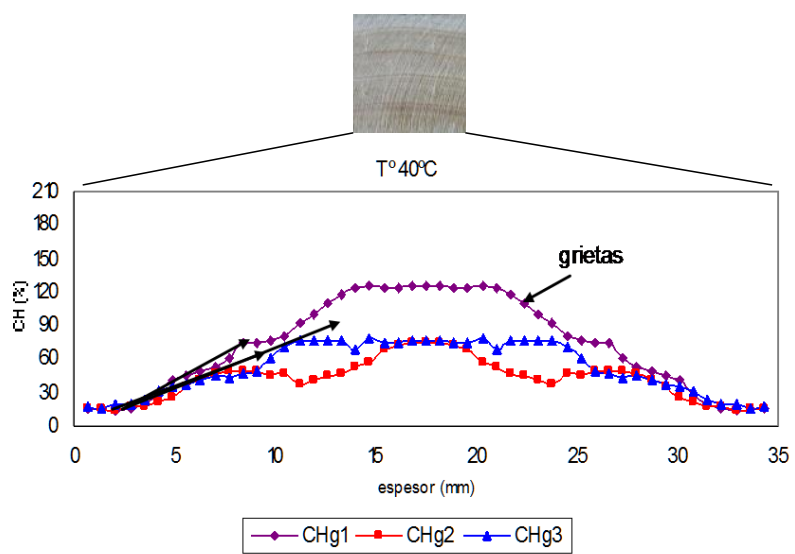

Figura № 4

PERFIL DE HUMEDAD EN MADERA TEMPERATURA DE SECADO DE 40ㄷ

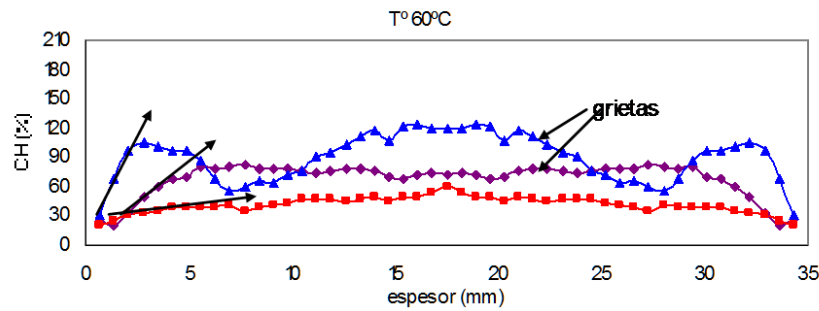

$\longrightarrow \mathrm{CHg} 1 \longrightarrow \mathrm{CHg} 2 \rightarrow \mathrm{CHg} 3$

Figura № 5

PERFIL DE HUMEDAD EN MADERA TEMPERATURA DE SECADO DE 60ㄷ

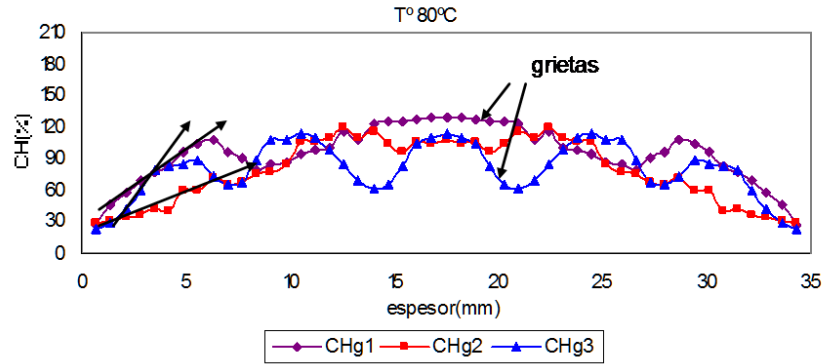

Figura № 6

PERFIL DE HUMEDAD EN MADERA TEMPERATURA DE SECADO DE 80ㄷ 


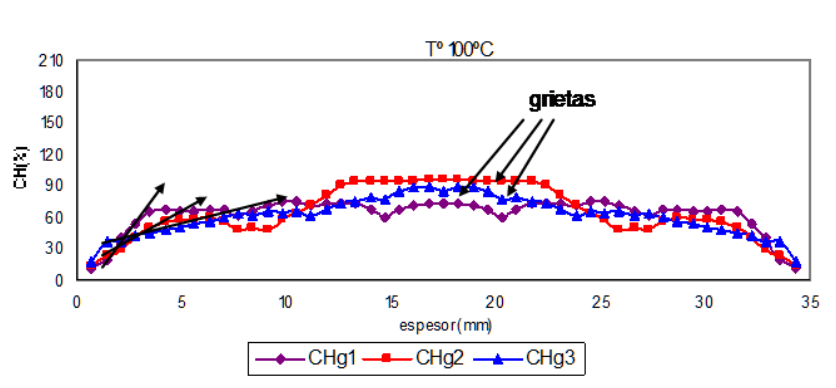

Figura № 7

PERFIL DE HUMEDAD EN MADERA TEMPERATURA DE SECADO DE $100^{\circ} \mathrm{C}$

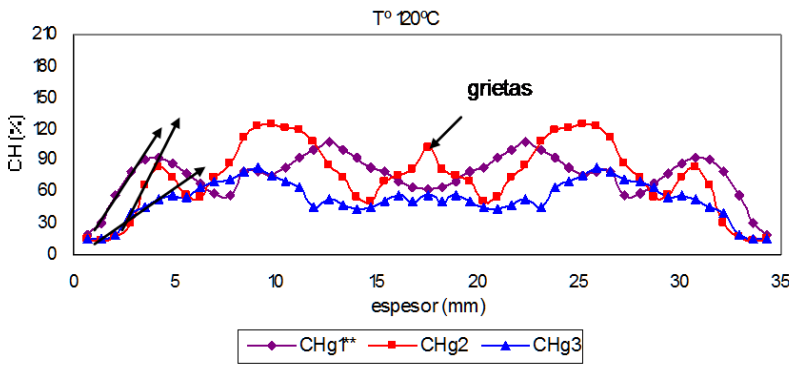

Figura № 8

PERFIL DE HUMEDAD EN MADERA TEMPERATURA DE SECADO DE 120ㄷ

Se puede observar que existe una leve tendencia de aumentar el perfil de humedad al aumentar la temperatura, esto significa que al aplicar temperaturas muy elevadas la superficies de las muestras perderán humedad mucho mas rápido alcanzando un contenido de humedad cercano o bajo al PSF, mientras que en el centro el contenido de humedad todavía se encuentra sobre el PSF.

En general, los perfiles más altos determinados entre la superficie y el centro están relacionados con la aparición de grietas superficiales, independientemente de la temperatura empleada. Es decir, donde se obtuvieron los gradientes más altos hubo presencia de grietas.

La temperatura de $40^{\circ} \mathrm{C}$ presentó la mayor diferencia de humedad entre la superficie y el centro, con un contenido de humedad de $14,9 \%$ en la superficie y $126,4 \%$ en el centro, y el menor valor de gradiente de humedad se aprecia en la muestra 2 a la temperatura de $60^{\circ} \mathrm{C}$, con una humedad de $19,9 \%$ en la superficie y $59,0 \%$ en el centro.

En general, se puede apreciar que los mayores perfiles de humedad están relacionados con el mayor contenido de humedad inicial de las muestras.

Las curvas de perfil de humedad, sobre todo en las capas contiguas a la superficie, caracterizan a esta madera como poco permeable. Estas curvas se pueden comparar con las obtenidas por Rice and Young (1991), donde no existe movimiento de agua libre. 
De acuerdo con varios autores (Kollmann \& Cote, 1968; Siau, 1984) el movimiento de agua capilar ocurre de una célula a otra por las aberturas naturales que posee la madera. Por eso para las especies de Eucalyptus este movimiento es obstaculizado por las características anatómicas propias de esta especie; los diámetros de sus puntuaciones son pequeños y sus vasos se encuentran usualmente bloqueados por tilosis.

Estas características definen a esta especie como poco permeable, lo que hace que el flujo de masa con respecto al tiempo se dificulte.

\section{CONCLUSIONES}

El microtomo de desplazamiento horizontal facilita satisfactoriamente la obtención de láminas para determinar los perfiles de humedad, permitiendo acercarse al comportamiento real del perfil de humedad que existe en un determinado espesor.

Independientemente de la temperatura de secado, el comportamiento que adoptan los perfiles de humedad con respecto a su espesor es similar, en la superficie se aprecia un contenido de humedad bajo el PSF mientras que en el centro de la pieza el contenido de humedad esta sobre este punto.

Existe una estrecha relación entre el contenido de humedad inicial de la madera y el contenido de humedad en el cual aparecen las grietas superficiales.

En lo referente a la permeabilidad que posee Eucalyptus nitens, esta es una especie poco permeable, lo que significa que existen mas obstáculos para la eliminación del agua libre y es por esto que las temperaturas aplicadas en las primeras etapas del proceso no deben ser mayores a $40^{\circ} \mathrm{C}$, ya que en esta etapa es donde se produce un rápido secamiento de la superficie lo que genera gradientes de humedad excesivos y hace posible la aparición de grietas superficiales.

\section{REFERENCIAS}

De Andrade, A., 2000. Indicação de programas para secagem convencional de madeiras. Teses de Magister, Universidad de São Paulo, Piracicaba, 72p.

Galvão, A. P. M. y Jankowsky, I. P., 1985. Secagem Racional da Madeira. São Paulo: Nobel, 111 p.

Hart, A. C. and Darwin, M. W., 1971. The slow drying rate of White Oak. Wood Science, 4 (1): 46-54.

INN, 1986a. Norma Chilena Oficial 176. NCh 176/1. Determinación del contenido de humedad de la madera. Primera Edición. Santiago Chile.

INN, 1986b. Norma Chilena Oficial 176. NCh 176/2. Determinación de la densidad de la madera. Primera Edición. Santiago Chile.

Kollmann, F. F. P. and Côté, W. A., 1968. Principles of wood science and technology solid wood. New York: Springer, v.1, $592 \mathrm{p}$.

Resch, H.; Kang, H. and Hoag, L. M., 1989. Drying Douglas Fir lumber: a computer simulation. Wood and Fiber Science. 21 (3): 207-218.

Rice, W. R. and Young, L. R., 1991. One and two dimensional moisture profiles in Red Oak. Wood and Fiber Science, Madison, 23 (3): 328-341.

Rozas, C. y Gallardo, R., 2003. Variación de la densidad básica con la altura del árbol en Eucalyptus nitens y globulus de 10 y 14 años de edad. CIS-MADERA. No 10 -1er semestre: 67-76.

Rozas, C.; Sanchez, R. y Pinedo, P., 2005. Secado de Eucalyptus nitens y E. globulus y su aprovechamiento en 
blocks, pisos y muebles. Maderas, Ciencia y Tecnología. 7(2):109-120.

Rozas, C., 2007. Modelo de transferência de calor y massa na secagem de madeira serrada de Pinus. Tesis de Doctorado, Universidad Fedral de Paraná, Curitiba, Brasil. 155 p.

Siau, J. F., 1984. Transport processes in wood. New York: Springer-Verlag, 245 p.

Simpson, W. T., 1976. Effect of presteaming on moisture gradient of Northern Red Oak during drying. Wood Sci., Madison, 8: 156-159. 